\title{
An observational study on morphology of azygos vein
}

\author{
Maruthy Prasad \\ Assistant Professor, Dept. of Anatomy, DM Wayanad Institute of Medical Sciences, Wayanad, Kerala, India \\ *Corresponding Author: Maruthy Prasad \\ Email: drmaruthy75@gmail.com
}

Received: $23^{\text {rd }}$ July, 2018

Accepted: $7^{\text {th }}$ August, 2018

\begin{abstract}
Introduction: Azygos system of veins comprising unpaired azygos vein and its main tributary, the hemiazygos veins. Variations have been observed in formation, course of azygos vein, also in its, tributaries and anastomoses.

Objectives: To observe azygos venous system pattern.

Materials: 50 embalmed human cadavers irrespective of their sex and age.

Methods: Following Anson's classification system in which azygos venous system having vertical and horizontal connections which include three basic types, primitive or embryological types, transient type, and single type as and their eleven subgroups.

Results: The basic type pattern in all the specimens (100\%) was transitional type. In subgroups: atypical type observed in 12 specimens (24\%). group 5 \& group 6B was observed in 11 specimens (22\%), group 2 was observed in five specimens (10\%), group 4 was observed in five specimens (10\%), group 7 was observed in 3 specimens (06\%), group 10 was observed in 03 specimens $(06 \%)$.

Conclusion: The importance's of variations in the pattern of azygos venous system which has to be identified during computed tomography and magnetic resonance imaging of mediastinum may be confused with aneurysm, lymphadenopathy and other abnormalities like tumor. During surgery of large vessel and the mediastinal operations, these kinds of azygos venous system pattern variations have to keep in the mind.
\end{abstract}

Keywords: Azygos vein, Morphology, Azygos venous system types.

\section{Introduction}

The azygos system of veins consists of a series of longitudinal vessels on each side of the body that drain blood from the body wall and move superiorly to empty into the superior vena cava \& serves as an important anastomotic pathway of returning venous blood from the lower part of the body to the heart if inferior vena cava is blocked. Blood from some of the thoracic viscera may also enter the system and there are anastomotic connections with abdominal veins. The major veins in the system are the azygos vein, on the right and the hemiazygos vein and the accessory hemiazygos vein, on the left. ${ }^{1}$ the thoracic wall and upper lumbar region are drained by the posterior and lumbar veins into azygos system of veins. ${ }^{3}$ There is a significant variations in the origin, course tributaries, anastomoses and termination of these vessels. ${ }^{1}$ Retro aortic transvertebral connections from hemiazygos and accessory hemiazygos vein to the azygos vein are also variable: there may be up to five connections. ${ }^{2}$

To observe and document the variations in the pattern of the azygos venous system in human cadavers was the aim of study which may be helpful in reducing misinterpretations in CT \& MRI imaging of mediastinum as some anomalies of the azygos venous system which can be easily confused with pathological conditions such as aneurysms, tumours and enlarged lymph node. ${ }^{4}$ Further to prevent errors during the surgery of the posterior mediastinum, surgeons should be aware of possible variations in the azygos venous system. Not many studies have been done on azygos venous system variations. Hence the study became essential.

\section{Materials and Methods}

Observational study on the pattern of azygos venous system in 50 embalmed human cadavers irrespective of age and sex which were allotted for medical undergraduates in the Departments of Anatomy, Kempegowda Institute of Medical Sciences, Bangalore Medical College and Research institute, MS Ramaiah Medical College, Bangalore, Mysore Medical College and Research institute, Mysore, and Mandya institute of Medical sciences, Mandya, between 2011 and 2013.

Inclusion Criteria: Adults cadavers irrespective of age $\&$ male or female.

Exclusion Criteria: New born, infants and children and Cadavers with scar over the chest.

Methods: According to the guidelines of Cunningham's manual of practical anatomy, volume two, Thorax and Abdomen fifteen editions, anterior thoracic wall was removed and the pericardium, heart, lungs, thoracic aorta and esophagus were removed from the thoracic cavity and the posterior intercostal veins were exposed. ${ }^{5}$ following the posterior intercostal veins course of right side, the azygos vein was traced. The hemiazygos vein and accessory hemiazygos veins were traced by following the course of left posterior intercostal veins. The transverse communication between azygos vein, hemiazygos vein and accessory hemiazygos veins was also noted. Following Anson BJ 
study, on the basis of the vertical forms and horizontal connections of azygos venous system, cadavers were divided into three major types of azygos venous system (primitive or embryological or double type, transitional type and single column type), again these three types were subdivided in 11 subtypes. After observation, the moisture over the vein and nearby areas was removed with filter paper. Numbering and photography were done duly for all the specimens.

\section{Statistical Analysis}

Data was analysed by using SPSS 4 windows.

\section{Results}

In present study, transitional major group type pattern of azygos venous system was observed in all the 50 specimens $(100 \%)$.

In subgroups; atypical group was observed in 12 specimens (24\%) followed by group $5 \&$ group 6B azygos venous system pattern was observed in 11 specimens (22\%). Group 4 \& group 2 azygos venous system pattern was observed in 5 specimens (10\%), group $7 \&$ group 10 pattern of azygos system in 3 specimens $(6 \%)$ \& observations of group 3 azygos venous system pattern in 1 specimen $(2 \%)$.

Observation of group1, group 3, group 6A, group 8 , group $9 \&$ group 11 pattern of azygos venous system were not found.
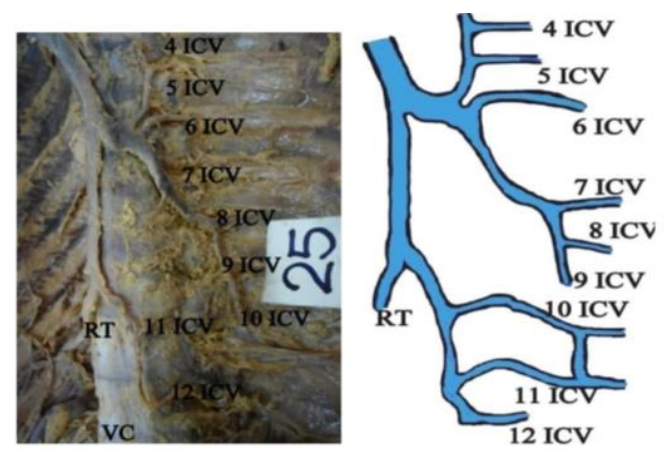

Fig. 1: Diagrammatic representation of specimen no. 25
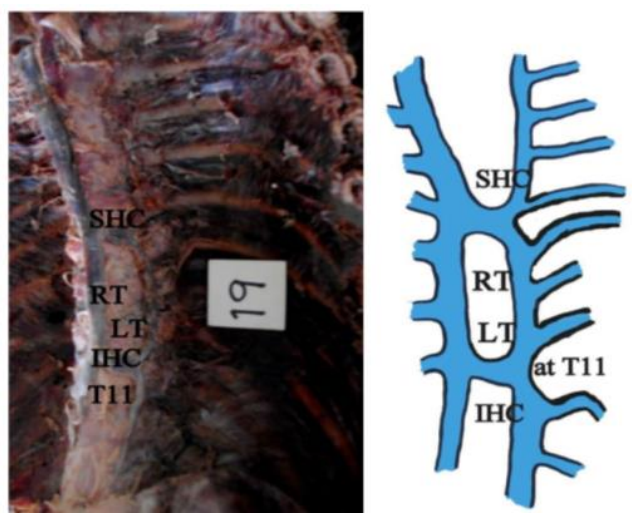

Fig. 2: Diagrammatic representation of specimen no.
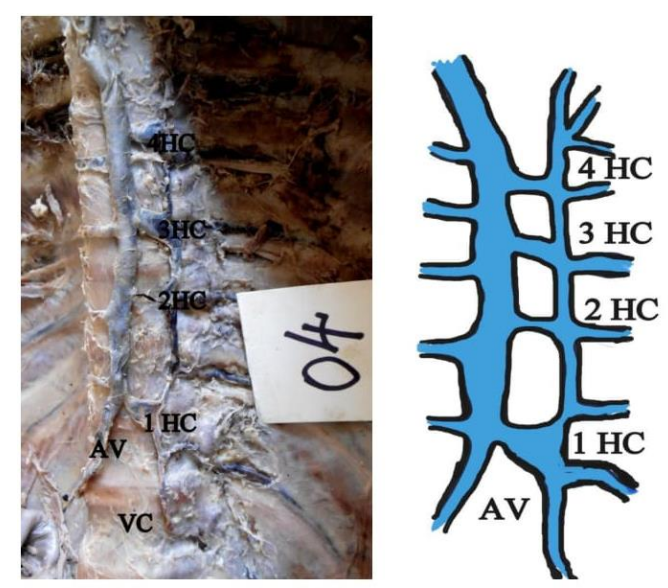

Fig. 3: Diagrammatic representation of specimen no. 4

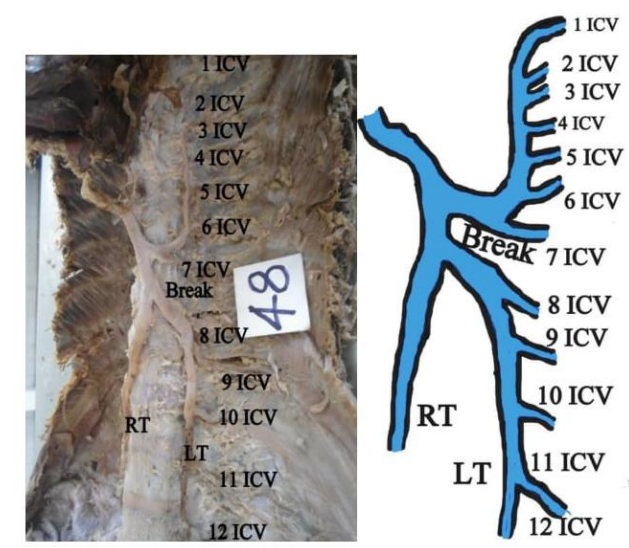

Fig. 4: Diagrammatic representation of specimen no. 48
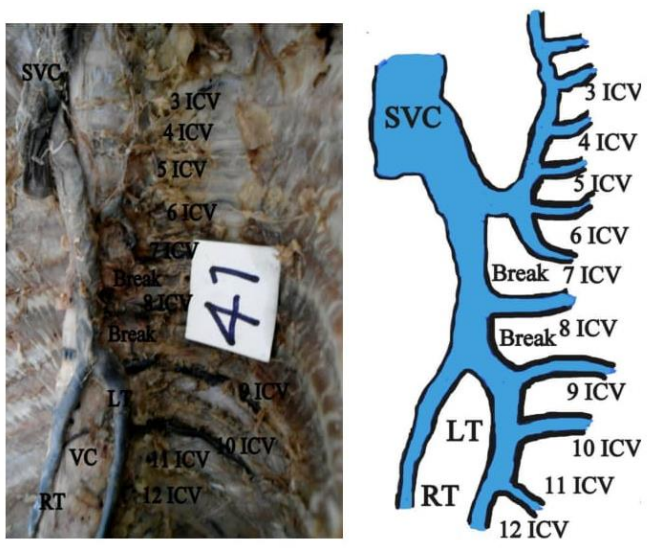

Fig. 5: Diagrammatic representation of specimen no. 41 


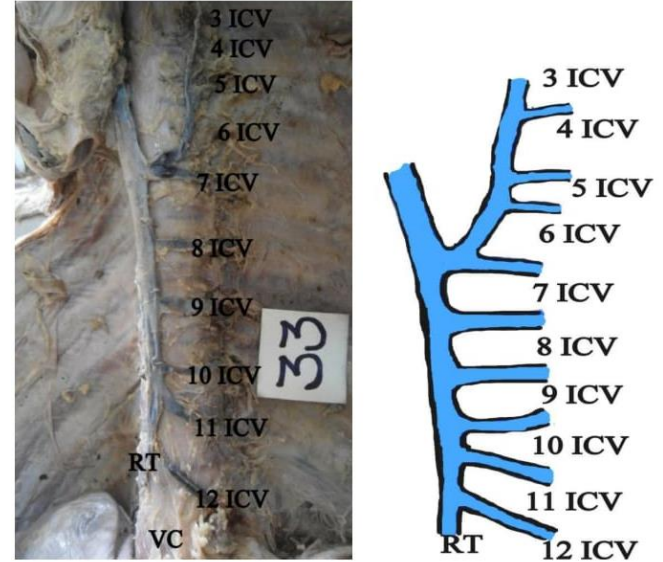

Fig. 6: Diagrammatic representation of specimen no. 33

\section{Discussion}

Azygos venous system pattern was broadly classified into 3 major group's types; double, transitional and single type according to the classification by Anson BJ et al; ${ }^{6}$

1. Two separate, parallel vessels lying along the lateral anterior border of the vertebral column in the posterior mediastinum forms 'primitive or embryological or double' type of azygos venous system.

2. A series of gradations form a pattern having a single communication between the azygos and hemiazygos systems to multiple retro aortic communications forms 'transitional' type of azygos venous system.

3. A single azygos vein occupying the midline of the anterior surface of the thoracic vertebra forms 'single' type of azygos venous system. ${ }^{6}$

Double type of azygos venous system was observed in 58\%, transitional type of azygos venous system was observed in $37 \%$ and observation in 5\% was single type of the azygos venous system. Double type of azygos venous system was significantly predominant in the American whites: the single type was predominant in American Negroes. ${ }^{8}$

Double type of azygos venous system was observed in $2.1 \%$, transitional type of azygos venous system was observed in $95.8 \%$ and observation of $2.1 \%$ was single type of the azygos venous system. ${ }^{6}$

In present study, transitional type of azygos venous system was observed in $100 \%$, double type \& single type of azygos venous system was not observed $(0 \%)$.

Further major types were sub classified into 11 Groups with according with Anson BJ classification of the pattern of azygos venous system as follows:

Group 1: Two completely separate, parallel ascending veins: the azygos on the right, the inferior and superior azygos on the left in continuity, this pattern type of azygos venous system was not seen in the present study.
Group 2: One midline horizontal connection usually at the level of T8, between right and left trunk, which was observed in 5 specimens (10\%).

Group 3: Between right and left trunk, one horizontal connection above the level of T8, this pattern of azygos venous system was not observed in the present study.

Group 4: Two horizontal connections between right and left trunk superior horizontal connection at T8 and inferior horizontal connection below the level of T8, which was observed in 5 specimens (10\%) (Fig. 2).

Group 5: Three to five horizontal connections between right and left trunk, which were observed in 11 specimens (22\%) (Fig. 3).

Group 6: One breakage in Left trunk, again this group is divided into i) Group 6A: AHazv and left brachiocephalic trunk vein is connected. ii) Group6B: No connection between AHazv and left brachiocephalic trunk vein. In the present study, group 6A pattern was not observed. Group 6B pattern was observed in 11 specimens (22\%) (Fig. 4).

Group 7: Two breakages in left trunk which was observed in 3 specimens (06\%) (Fig. 5).

Group 8: One break in the left trunk, above and below the break; posterior intercostal veins are joined by collaterals.

Group 9: Five breakages in the lower part of the left trunk, and two single veins, in the lower part formed by joining of $11^{\text {th }}$ and $12^{\text {th }} \mathrm{ICV}$ and $10^{\text {th }}$ and $9^{\text {th }} \mathrm{ICV}$. Group 8 and Group 9 patterns were not observed in this study.

Group 10: Five breakages in the lower part of the left trunk which was observed in 3 specimens (06\%) (Fig.: 6).

Group 11: Single azygos vein occupying the midline of the anterior surface of the thoracic vertebra. In the present study this pattern of with this pattern of azygos system was not observed.

Atypical Group: Azygos venous system pattern which was not fulfilling the above mentioned criteria was observed in 12 specimens (24\%), (Fig. 1).

In subtypes, group 2 was observed in $27.11 \%$ followed by group 7 in $22.9 \%$ group $4 \&$ group 5 in $10.4 \% .^{6}$ Where as in present study, atypical group formed majority group which was observed in $24 \%$, other prevalent groups were group $5(22 \%)$ and group 6B $(22 \%)$. Variations in pattern of azygos vein are prevalent as atypical group was prominent in the present study.

Embryology: For convenience and apparent simplicity, early embryonic veins are segregated as visceral and somatic group. The visceral group includes the derivates of vitelline and umbilical veins and the somatic group includes all the remaining veins. ${ }^{2}$ Azygos line veins are one the of components of somatic group veins which are formed dorsolateral to the aorta and medial to the sympathetic trunk, also referred as medial sympathetic line veins gradually take over the intersegmental veins drainage from the supracardinal 
veins. Cranially, the azygos lines join the persistent ends of the posterior cardinal veins. ${ }^{2}$

The vertical part of the azygos vein is formed by right azygos venous line, the arch of azygos vein is formed by persistent of the cephalic part of the right posterior cardinal vein. The left azygos venous line forms the superior and the inferior hemiazygos veins. ${ }^{10}$ Subcentral veins (transitory longitudinal vessels) form directly dorsal to the aorta in the interval between the origins of the paired intersegmental arteries communicate freely with each other and with the azygos line vein; these connections ultimately form the retro-aortic parts of the left lumbar veins and of the hemiazygos veins. ${ }^{2}$ Persistence above mentioned communications may have been observed in the present study.

The communication of the subcentral vein with the azygos line normally takes place posterior to the aorta. ${ }^{4}$ With decrease in importance of the left common cardinal vein the blood in the left azygos system, below the level of the third left intercostal vein, drains across the middle line behind the aorta to the right azygos system which, in turn, drains into the persistent cephalic termination of the right posterior cardinal. ${ }^{7}$

In CT imaging of posterior mediastinum, a retroaortic anastomosis are sometimes large enough even without major vein obstruction, to be mistaken for an enlarged lymph node may be due to obstruction of superior or inferior vena cava, azygos or hemiazygos vein continuation of right or left inferior vena cava or portal hypertension may dilate the azygos, hemiazygos vein and their retro-aortic anastomosis as collateral pathways. ${ }^{10}$

\section{Conclusion}

Present study demonstrated variations in pattern of azygos venous system which may help in reducing the misinterpretation of the CT \& MRI appearance of hemiazygos and accessory hemiazygos vein and retroaortic anastomoses as normal or enlarged lymph node in pathological conditions. Further awareness about possible variations observed in pattern of azygos venous system may help surgeons during surgeries involving thoracic vertebrae, large vessels of posterior mediastinum.

\section{Abbreviations}

HC - Horizontal connection: ICV - Inter costal vein

LT -Left trunk:

SHC-Superior horizontal

connection:

RT- Right trunk

horizontal connection

T11-Eleventh thoracic vertebra: AV- Azygos vein,

VC-Vertebral column: SVC- Superior vena

cava

\section{References}

1 Richard LD, Wayne AV, Adam WMM, GREY'S Anatomy for students. Second edition (International edition). Canada: Elsevier Churchill Livingstone; 2010, 218p.

2 Susan S, Neil RB, Patricia C, et al. GREY'S Anatomy the Anatomical Basis of Clinical practice. Fortieth edition (International edition). China: Elsevier Churchill Livingstone; 2008;940:207.

3 Chummy SS .Last's Anatomy, Regional and applied. $12^{\text {th }}$ edition. China: Elsevier Churchill Livingstone, 2011, 211p.

4 Ozbek A, Dacik k, Colak T. Multiple variations of the azygos venous system. Surgical Radiology Anatomy. 1999;21:83-85.

5 Romanes GJ. Cunningham's manual of practical Anatomy vol 2.15th edition. London: Churchill Livingston: $1986: 75 p$

6 Kutuglu T, Turut M, Kocabiyik. Anatomical analysis of azygos vein system in human cadavers. Romanian Journal of Morphological and Embryology. 2012;53(4):1054-1056.

7 Hamilton, Boyd, Mossman. Human Embryology prenatal development of form and function. Fourth edition New York: the Macmillan press 1td; 1976:277-280.

8 George AS. "The Azygos system of veins in American whites and American Negros including observation on the inferior vena cava" American Journal of Physical Anthropology. 1934;XIX.1:156.

9 Datta AK. Essential of Human embrology. $6^{\text {th }}$ edition. Calcutta: Current Books International, 2005:192-194.

10 Julie ET, David JG. CT appearance of the Retroaortic Anastomosis of the Azygos System. AJR. 1990;154:4144.

How to cite this article: Prasad M. An observational study on morphology of azygos vein. Indian J Clin Anat Physiol. 2018;5(4):497500 . 